\title{
The minimum distance diagram and diameter of undirected double-loop networks
}

\author{
Ying $\mathrm{Li}^{1, \mathrm{a}}$, Yebin Chen ${ }^{2,}$, Weipeng Tai ${ }^{2, \mathrm{a}}$, Renwei Wang ${ }^{2, \text { a }}$ \\ ${ }^{1}$ School of Science and Engineering, Ma'anshan Teacher's College, Ma'anshan Anhui, 243041, \\ China \\ ${ }^{2}$ School of Computer Science, Anhui University of Technology, Ma'anshan Anhui, 243002, China \\ acyb7102@163.com
}

\begin{abstract}
Keywords: undirected double-loop networks; minimum distance diagram; shortest path; optimal network.
\end{abstract}

\begin{abstract}
This thesis proposes a minimum distance diagram of undirected double-loop, which makes use of the cyclic graph's excellent properties on the node number and path length to achieve high performance. Main issues include the method to build the minimum distance diagram of undirected double-loop networks, the algorithm to calculate its diameter, and the step to build optimal undirected double-loop networks, etc. We prove that the diameter of the undirected double-loop network is equal to the height of its tree structure, and propose a rapid algorithm to calculate the diameter, and find that there are lots of optimal undirected double-loop networks in some infinite clusters. Finally, the lower bound proposed by Yebra is verified by experiment. According to these results above, the transmission performance of undirected double-loop will be optimized.
\end{abstract}

\section{Introduction}

The double-loop network which has symmetry, simple structure, easy scalability and strong fault tolerance, is easy to be constructed and widely used. High reliability of double-loop Networks is closely related to the network transmission delay, which is relevant to the network diameter and its routing algorithm, the shorter diameter brings about the less transmission delay, and the better routing algorithm will get the shortest path between any two nodes. So, how to choose the proper steps to decrease the diameter, how to design the optimal routing algorithm, these issues are worth to study.

According to the principle of communication that shorter diameter means less delay. Suppose an undirected double-loop network $G(N ; \pm r, \pm s)$ has a limited diameter $\mathrm{d}(N ; \pm r, \pm s)$, denote $\mathrm{D}(N)=$ $\min \{\mathrm{d}(N ; \pm r, \pm s): 1 \leq r \neq s<N\}$, Wong et al. [1] gave the lower bound of $\mathrm{D}(N)$, that is $\mathrm{D}(N)$ $\geq(\sqrt{2 N}-3) / 2$. Later, Yebra et al. [2] adjust $\mathrm{D}(N) \geq[(\sqrt{2 N-1}-1) / 2]$. If $\mathrm{D}(N)$ obtains the minimum value (the lower bound), then we call $\mathrm{G}(N ; \pm r, \pm s)$ as optimal undirected double-loop network. A problem is that for a double-loop network with given $N$, how to calculate its diameter according to step $r$ and $s$ ? In this field, Chen [3-5] and Fang et al. [6-8] have done a lot of work and have made plenty of valuable achievements. Our further study is on the basis of results which researched by Chen et al. who had described the diameter upper bound of the undirected network $G(N ; \pm 1, \pm s)$, they also presented the formula of diameter about the undirected double-loop network $G(N ; \pm 1$, $\pm s)$, which can compute the diameter of undirected double-loop network whose the first step is 1, but it did not resolve the information routing problem, the same applies to Fang, they are not universal. The method of this thesis is mapping the space structure of the undirected double-loop network to a plane tree structure and gives its minimum distance diagram, according to witch, we not only can compute its diameter but also can find the shortest path between any two nodes rapidly. 


\section{The minimum distance diagram}

Undirected double-loop network graph theory model is an undirected graph $G(N ; \pm r, \pm s)$, whose each vertex $i$ is denoted as $0,1, \ldots, N-1$, and there are four undirected edges $i \rightarrow i+r(\bmod N), i$ $\rightarrow i+s(\bmod N), \quad i \rightarrow i+N-r(\bmod N)$ and $i \rightarrow i+N-s(\bmod N)$ denoted as [+r] edge, [+s] edge, $[-r]$ edge and $[-s]$ edge respectively, $r$ and $s$ are two natural numbers, and satisfy $1 \leq r \neq s<N$. Fig. 1 shows the topology of undirected double-loop network $\mathrm{G}(14 ; \pm 3, \pm 4)$. For a given $N, r$ and $s$ can decide the structure of the undirected double-loop networks $G(N ; \pm r, \pm s)$, it also can determines its diameter and routing strategy.

According to the symmetry of the double-loop network, the distance of node $u$ to node $v$ equals to the distance of node 0 to node $v-u$, therefore, we just pay close attention to routing strategies from node 0 to other nodes, and then we can know the whole routing table about this network. According to the above principles, the spatial structure of the undirected double-loop network $G(N$; $\pm r, \pm s$ ) can be mapped plane structure as Definition 1 .

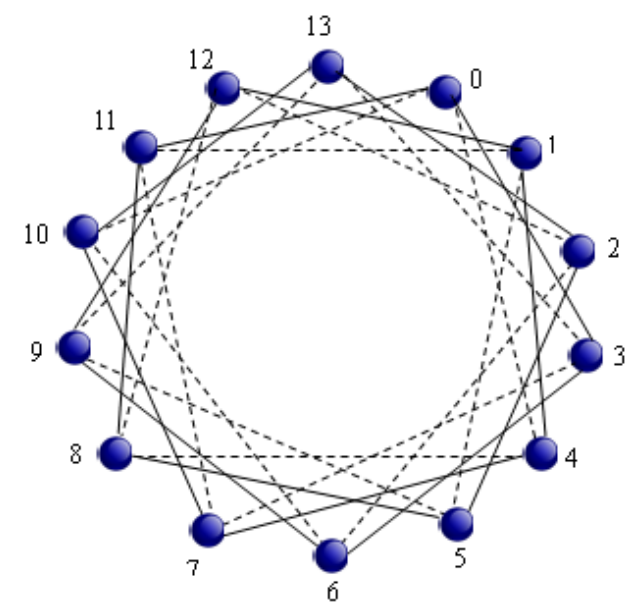

Fig. 1 The topology of undirected double-loop network $G(14 ; \pm 3, \pm 4)$.

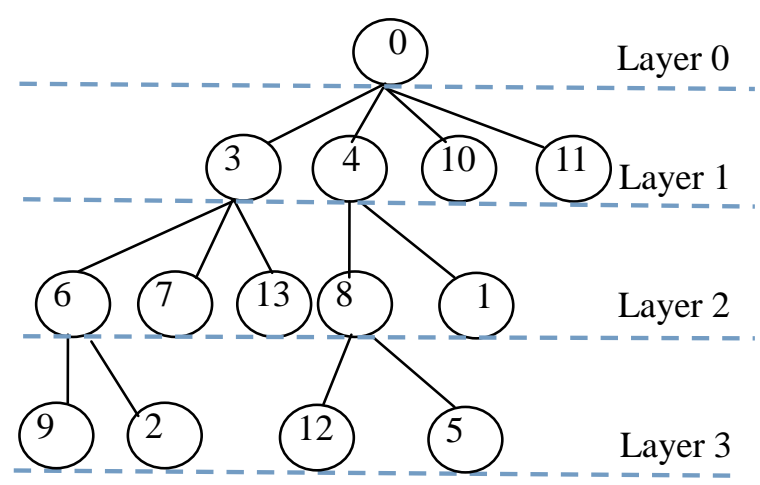

\section{Definition 1}

Fig. 2 The tree structure of undirected double-loop network $T(14 ; \pm 3, \pm 4)$.

Step1. Let node 0 as the root node of a tree put node 0 on layer 0 , write it and its child nodes $r, s$, $N-s, N-r$ to set $U$, put them on layer1 and construct the tree with five nodes as Fig.2 ;

Step2. Take each child node $i$ respectively to create its child nodes with the value of $i+r$ (mod $N), i+s(\bmod N), \quad i+N-r(\bmod N)$ and $i+N-s(\bmod N)$, if new child node have not appear in set $U$, then put it into $U$, otherwise abandon it, put these new nodes on layer 2 and construct child tree with these new nodes;

Step3. Repeat Step 2 above until all nodes appears in this tree.

The corresponding tree structure created by Definition 1 is denoted as $T(N ; \pm r, \pm s)$, which is the minimum distance diagram of undirected double-loop network. But no each $G(N ; \pm r, \pm s)$ has its $T(N$; $\pm r, \pm s$ ) beside gcd $(N, r, s)=1$. 
As you can see in Fig. 2, the different step can create different tree to the same node $N$, so the different step also get different diameter to the same node $N$. How to make messages transfer more efficiently in double-loop network systems? This problem has been received considerable attention, and a great number of results have been reported in [9-13]. They are mainly concerned with directed double-loop network, Chen et al. [9] presented the [+1]-link-prior routing algorithm which was considered more efficient than others, Fang et al. [10] believed that the efficiency of the [+h]-link-prior routing algorithm would be higher, Chen et al. [11-13] proved that both [+1]-link-prior or [+h]-link-prior are the same in efficiency. The routing features of undirected double-loop networks are the same with directed double-loop networks.

Fang et al. [14] studied the Cartesian coordinates by the minimum distance visiting mode forms an optimum graph $G(N ; \pm r, \pm s)$. By this graph according to whether source node and destination node lie in horizontal or vertical coordinate and the amount of fault nodes around them, the concepts of closing areas and escaping areas of faulty nodes are proposed. When escaping areas occur, there exists optimum routing paths from source node to destination node. Zhong Wei et al. [15] consider that the undirected double loop network $G(N ; \pm r, \pm s)$ is a Cayley graph with degree 4.The relationship between the four parameters of the $\mathrm{L}$ - shape tile and the smallest non-negative solution and the smallest cross solution of the congruence equation $x s_{1}+y s_{2}=0(\bmod N)$ is obtained. So the new diameter of $G(N ; \pm r, \pm s)$ can be represented by the four parameters of the L shape tile. For all these different structure above can be used to resolve different problem, but tree structure has some features that other structure is not equipped with.

\section{Main results}

Definition 2. For a tree structure $T(N ; \pm r, \pm s)$, define the layer root node in as 0 layer, define the layer its child nodes in as 1(first) layer, define the layer first layer's child node in as 2 layer, and so on.

Lemma 1. The shortest path length from node 0 to node $v$ is $l$, when the node $v$ is located in $l$ layer.

Proof. According to the Definition 1and Definition 2, node $v$ appears only once in $T(N ; \pm r, \pm s)$, and the distance from node 0 to node $v$ is $l$. Suppose there is a path is from node 0 to node $v$, its length is c, and c $<l$, then node $v$ must locate in c layer, it is incompatible with Definition 1. Therefore, the shortest path of node 0 to node $v$ is $l$.

Lemma 2. The necessary and sufficient condition starting from node 0 to node $v$ ( $0<v<N ; x, y$, $m, n \geq 0 ; x, y, m, n$ is positive integer) through $x[+r]$ edges, $y[+s]$ edges, $m[-r]$ edges and $n[-s]$ edges is $v=x r+y s+m(N-r)+n(N-s)(\bmod N)$.

Proof. Sufficient condition: since $v=(x r+y s+m(N-r)+n(N-s))(\bmod N)$, starting from node 0 through $x[+r]$ edges, $y[+s]$ edges, $m[-r]$ edges and $n[-s]$ edges, it will reach the node $v+k N$, as $v+k N(\bmod N)=v$, so it will reach node $v$.

Necessity condition: starting from node 0 through $x[+r]$ edges, $y[+s]$ edges, $m[-r]$ edges and $n$ $[-s]$ edges to node $v$, then $(x r+y s+m(N-r)+n(N-s))(\bmod N)=v+k N(\bmod N)=v$, we have $v=(x r+y s$ $+m(N-r)+n(N-s))(\bmod N)$.

Theorem 1. The shortest path length $l$ from node 0 to node $v$ is equal to the layer $c$ where the node $v$ located in.

Proof. Suppose $v \neq 0 \quad(U=\{0,1, \ldots, N-1\}), v=x_{1}[+r]+y_{1}[+s]+m_{1}[-r]+n_{1}[-s](\bmod N)$ $(0<v<N ; x, y, m, n \geq 0 ; x, y, m, n$ is positive integer $)$.

The path from node 0 to the node $v$ in 1 layer passes $1[+r]$ or $1[+s]$ or $1[-r]$ or $1[-s]$ when node $v$ located in $T(N ; \pm r, \pm s)$. Apparently, the shortest path length is $l={ }_{C}=1$.

When node $v$ located in the layer 2 in $T(N ; \pm r, \pm s)$, the path from node 0 to the second layer nodes are one of $2[+r]$ or $2[+s]$ or $2[-r]$ or $2[-s]$ or $1[+r]+1[+s]$ or $1[+r]+1[-r]$ or $1[+r]+1[-s]$ or $[+s]+[-r]$ or $1[+s]+1[-s]$ or $[-r]+[-s]$, obviously, the shortest path length is $l=c=2$.

Suppose node $v$ located in the $c$ layer, Theorem 1 is correct, that is $l=c$. When node $v$ located in 
the $c^{+1}$ layer, according to Definition 1 , the lower nodes of $T(N ; \pm r, \pm s)$ are their child nodes of the c layer, from $v$ to its child node only passes $1[+r]$ or $1[+s]$ or $1[-r]$ or $1[-s]$, so the path length from node 0 to node $v$ is the $c$ plus 1 , that is $l=c+1$.

According to the mathematical induction, the Conclusion set up.

Theorem 2.The diameter $d(N ; \pm r, \pm s)$ of the undirected double-loop network $G(N ; \pm r, \pm s)$ is equal to the height of $T(N ; \pm r, \pm s)$.

Proof. According to the definition of the diameter, the diameter $d(N ; \pm r, \pm s)$ of $G(N ; \pm r, \pm s)$ equals the maximum distance of root node 0 to any other nodes in $T(N ; \pm r, \pm s)$. The distance of root node 0 to nodes in the last layer is the maximum distance in $T(N ; \pm r, \pm s)$, so the diameter $d(N ; \pm r$, $\pm s)$ of $G(N ; \pm r, \pm s)$ is equal to the distance of root node 0 to nodes in the last layer. According to tree theorem, the height of tree is equal to the last layer of tree, so the diameter $d(N ; \pm r, \pm s)$ of the undirected double-loop network $G(N ; \pm r, \pm s)$ is equal to the height of $T(N ; \pm r, \pm s)$.

Definition 3. Given three parameters $N, r$ and $s(1 \leq r<s<N)$, let $r=1$; when the range of $s$ is from $r+1$ to $N-1$, we can get a group of undirected double-loop networks $G(N ; \pm r, \pm s)$ which is called an infinite cluster.

\section{The algorithm to calculate diameter}

Given $N, r$ and $s$ in a $G(N ; \pm r, \pm s)$, how to construct its corresponding tree structure rapidly is the chief problem to be settled. By Theorem 2, if we get the height of $T(N ; \pm r, \pm s)$, we also get the diameter of $G(N ; \pm r, \pm s)$, a rapid algorithm is provided as follow.

Algorithm. 1: Step 1. Initialization: For give $N, r$ and s, the root node is 0 , its child nodes are $r$, $s, N-r, N-s$, they are in the first layer, insert them into the linear list $l_{1}$, and put all these nodes into the set $U$, so $U=\{0, r, s, N-r, N-s\}$, let layer $=1, l_{2}$ is null;

Step 2. Get every node $i$ from the linear list $l_{1}$ in turn, construct its child node with the values of $i+r(\bmod N), i+s(\bmod N), i+N-r(\bmod N)$ and $i+N-s(\bmod N)$, if its child node have not appear in $U$, then put it into $U$, and insert them into the linear list $l_{2}$, otherwise do nothing, layer ++ ;

Setp 3. Delete all nodes from $l_{1}$, then insert all nodes from linear list $l_{2}$ to linear list $l_{1}$, empty the linear list $l_{2}$, repeat Step 2 until there is not any new node in linear list $l_{2}$;

Step 4. Output layer, which is the diameter $d(N ; \pm r, \pm s)$ of the undirected double-loop network $G(N ; \pm r, \pm s)$.

\section{Experimental results}

For these networks $G(N ; \pm r, \pm s)$ with same node $N$, there are different diameters $d(N ; \pm r, \pm s)$ for different step $r$ and $s$. For a $G(N ; \pm r, \pm s)$, if its diameter satisfies $d(N ; \pm r, \pm s)=l_{\mathrm{b}}(N)=\lceil(\sqrt{2 N-1}-1) / 2\rceil$

( $\lceil x\rceil$ is the smallest integer not less than $x$, it ) is called optimal network. How to find steps for optimal network? What is the distribution characteristics for these optimal networks and the $d(N ; \pm r$, $\pm s$ ) of an infinite cluster? All these answers will be found by simulation experiments. Some computer simulation and experiment results are presented in Fig. 3 (a), (b). 


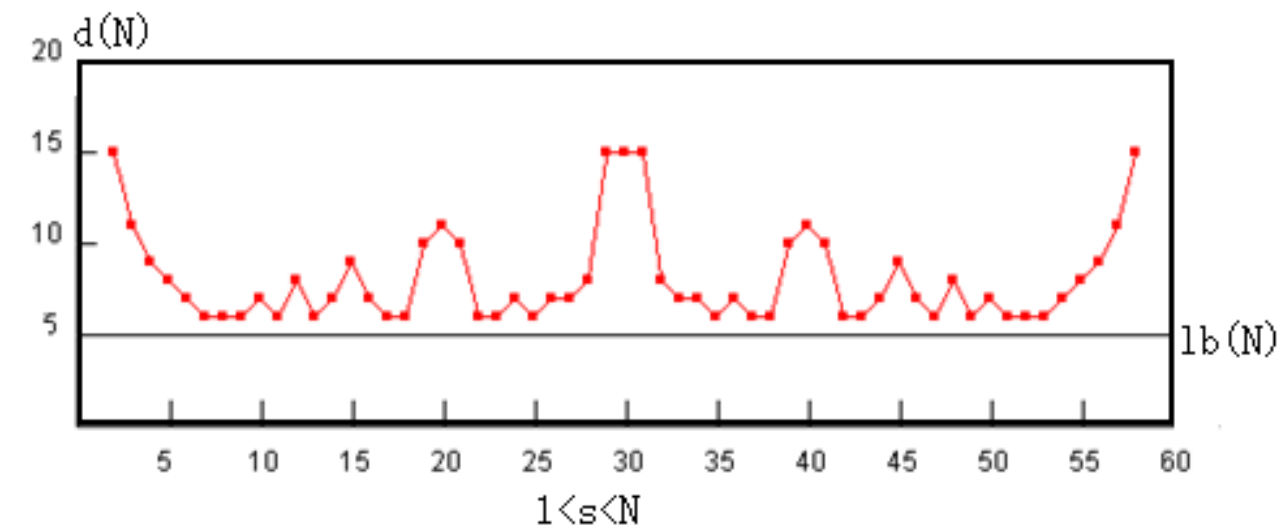

(a) $G(60 ; \pm 1, \pm s)$

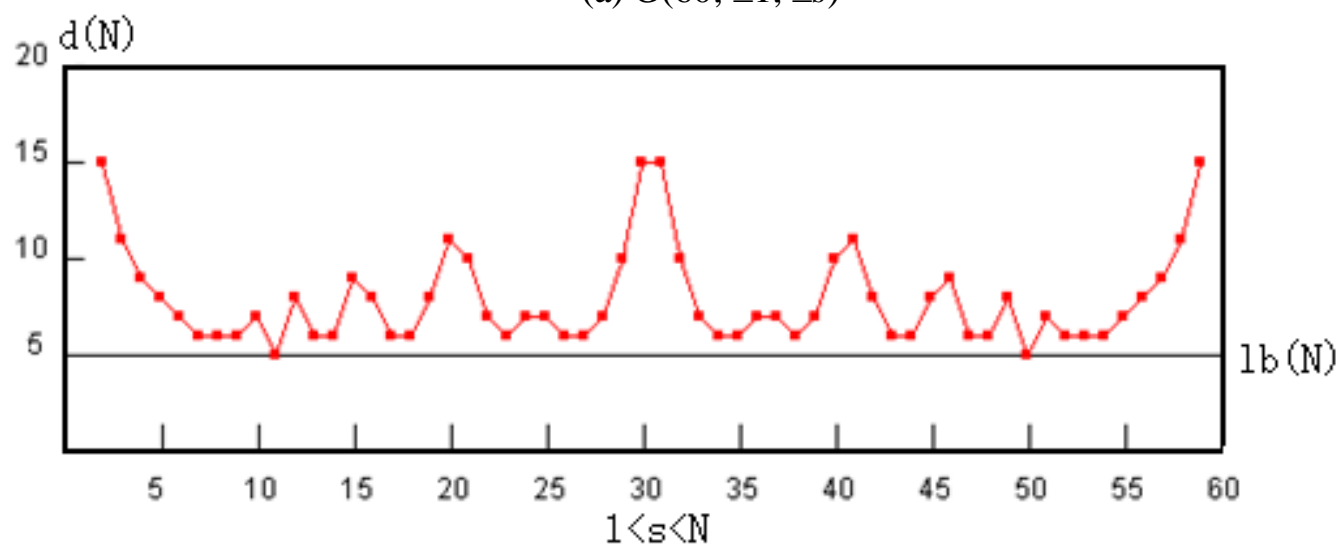

(b) $G(61 ; \pm 1, \pm s)$

Fig. 3 the distributions of diameters in infinite cluster

Fig. 3 (a), (b) represent some infinite clusters, e.g. $G(60 ; 1, s)$ and $G(61 ; 1$, s). Through these experiment results, we will verify some theories above and get more conclusions as follows:

(1) For any undirected double-loop network $G(N ; \pm r, \pm s)$, the conclusion $l_{\mathrm{b}}(N)=\lceil(\sqrt{2 N-1}-1) / 2\rceil$ proposed by Yebra is correct, see Fig. 3 (a) , (b), $l_{\mathrm{b}}(60)=5, l_{\mathrm{b}}(61)=5$.

(2) For any infinite cluster of undirected double-loop network $G(N ; \pm r, \pm s)$, the distribution of diameters is an axis-symmetrical figure, the maximums always located in the center of symmetry and two borders.

(3) There are two optimal networks in an infinite cluster, see Fig. 3 (b), such as $G(61 ; 1,10)$, $\mathrm{G}(61 ; 1,50)$, they always appear in pairs. But not all are like that; see Fig. 3 (a).

\section{Conclusions}

Double loop structure is not only good for most of the problems, but also their defects. This paper use undirected double-loop network in the node degree, network diameter and optimal networks properties etc. Firstly we studied its topology and mapped it to tree structure, so we got the minimum distance diagram, and then we got related algorithm about diameter, finally we found some optimal networks in an infinite cluster through experimental result. But how to get suitable steps to construct optimal network, what is the distribution characteristics for these optimal networks, these problems will be the primary problems for we to resolve.

\section{Acknowledgments}

This research was supported by the Natural Science Important Project of Anhui Province Education Department under contract KJ2015ZD39 and Professional leaders of Anhui Province in higher vocational college under contract 2014SDTR34. Sincere thanks are due to those anonymous referees who provided unusually detailed and very helpful comments that improved the accuracy 
and clarity of our presentation.

\section{References}

[1] C.K. Wong, D. Coppersmith, A combinatorial problem related to multimodule memory organizations, Journal of the ACM, 21 (1947): 392-402.

[2] J.A.L. Yebra, M.A. Fiol, P. Morillo, et al. The diameter of undirected graphs associated to plane tessellations[J]. Ars Combinatoria, 20B(1985): 151-171.

[3] B.X. Chen, W.J. Xiao, X.N. Huang. Estimations for Diameters of Undirected Double-Loop networks[J].Journal of ZhangZhou Teachers College(Nat.Sci.), 48(2005): 7-13.

[4] B.X. Chen, W.J. Xiao, P. Behrooz. Diameter Formulas for a class of Undirected Double-Loop Networks[J]. Journal of Interconnection Networks, 6(2005): 1-15.

[5] B.X. Chen, J.X. Meng, W.J. Xiao. Some new optimal and suboptimal infinite families of undirected double-loop networks. Discrete Mathematics and Theoretical Computer Science, 8(2006): 299-312.

[6] M.Y. Fang, B.H. Zhao, Y.G. Qu, et al. A new method to calculate the diameter of undirected double- loop network. Journal of Huazhong University of Science and Technology (Natural Science Edition), 34 (9) (2006): 14-17.

[7] M.Y. Fang, B.H. Zhao. Method to calculate the diameter of undirected double-loop network $\mathrm{G}(\mathrm{N} ; \pm 1, \pm \mathrm{s})$. Journal on Communications, 28 (2) (2007): 124-129.

[8] M.Y. Fang, H.Z. PENG, H. LIU. Research on fault-tolerant routing of bidirectional double-loop networks. Computer Engineering and Applications, 49(14)(2013): 105-108.

[9] Z.X. Chen, J. Fan, On the [+1] link-prior shortest path and optimal routing for double-loop networks, Journal of Computer Research \& Development, 38 (7) (2001): 788-792.

[10] M.Y. Fan, Y.G. Qu, B.H. Zhao, [+h] link prior routing strategy for double-Loop network, Chinese Journal of Computers, 31 (3) (2008): 536-542.

[11] Y.B. Chen, Bintree-based shortest path algorithm of directed double-loop networks, Journal of Huazhong University of Science and Technology (Natural Science Edition), 37 (4) (2009) : 78-81.

[12] Y.B. Chen, Y. Li, T. Chen. Optimal fault-tolerant routing algorithm and fault-tolerant diameter in directed double-loop networks. Theoretical Computer Science, 468(2013): 50-58.

[13] Y.B. Chen, Y. Li, J.k. Wang. On the Wide Diameter of Directed Double-loop Networks. Journal of Network and Computer Applications, 34(2011):692-696.

[14] ZH. Wei, B.X. CHEN, S.Q. ZHU. New diameter formula for undirected double-loop network. Computer Engineering and Applications, 2010, 46(32):84-87.

[15] M.Y. FANG, H.Z. PENG, H. LIU. Research on fault-tolerant routing of bidirectional double-loop networks. Computer Engineering and Applications, 2013, 49(14):105-108. 